\title{
Application of yeast with reduced alcohol yield for sparkling wine production
}

\author{
M. Schmitt ${ }^{\mathrm{a}}$, S. Broschart, C.-D. Patz, D. Rauhut, M. Friedel, and D. Häge \\ Hochschule Geisenheim University von Lade Straße 1, 65366 Geisenheim, Germany
}

\begin{abstract}
Two commercial yeast strains with reduced alcohol production in comparison with a commercial yeast strain with common alcohol yield were assed for their suitability in sparkling wine production according to the traditional bottle fermentation. The different yeast strains were applied for the first fermentation. As expected the base wine differed in terms alcohol. Furthermore the yeast with lower alcohol content showed higher values of glycerol, higher arginine content and in the same time reduced levels of proline after fermentation. However those samples showed increased volatile acidity values, compared to the control wines. The later bottle fermentation with a uniform yeast strain showed similar fermentation kinetics for all four lots. Sensory evaluation showed no clear differences between the sparkling wines that were stored 9 months on the lees. The base wines nevertheless clearly differed from each other. Besides the increased production of volatile acidity, the tested yeast strains with lower alcohol production appear very promising for the sparkling wine industry to face the generally rising alcohol contents worldwide.
\end{abstract}

\section{Introduction}

Various sources show increasing alcohol levels for wine within the last decades. The driving factors for elevated alcohol levels can be seen in improved viticultural practices, advanced plant material, optimized yeast in combination with rising temperatures due to climate change.

Elevated alcohol levels affect the sensory characteristics of wine and sparkling wine in a very complex way. The influence of ethanol in wine is controversial and very complex. Alcohol has gustatory, olfactory and trigeminal stimulating properties $[10,15,22,29]$.

The direct sensory influence of alcohol is sweetness and the higher the alcohol content of the wine, the more the sweet perception increases $[15,19,27,30]$. Acid perception is reduced with increasing alcohol content [6]. To a certain extent, alcohol produces a sensation of warmth in the wine, which causes a particular burning sensation at excessive alcohol contents $[8,9]$.

Alcohol gives the wine body and fullness [8,20,21,24]. At what level of alcohol the sensation of body and fullness increases, depends on the wine matrix. With increasing ethanol content, the bitterness of the wine increases $[6,11$, $15,19]$.

Sparkling wine should have a fresh character and bitterness as well as burning sensations are seen as negative traits. Furthermore elevated alcohol levels make the second fermentation stressful and unsecure. So the base wine for later sparkling wine production should have an alcohol content of 10 to max. $12 \%$ vol. [2,7]. Otherwise in is unsure to achieve a complete and homogeneous second fermentation.

a e-mail: Matthias.Schmitt@hs-gm.de
Alcohol reduction by physical methods is legal in many countries worldwide and the different technologies are practicable. When these technologies are applied in a correct manner the quality is not affected severely [25] and $2 \%$ vol. alcohol reduction cannot be detected by trained panelists in sensory evaluations from the untreated control $[3,5,12,16,25]$.

Nevertheless these technologies are more or less expensive and mean an extra effort in winemaking. In some countries such a technical intervention effects, besides enological rules, regulations for spirit production.

The regulations for organic wine production in the European Union according to the EU regulation 203/2013 also do not allow an alcohol reduction by physical methods.

The common way of facing elevated alcohol levels for sparkling wine is to harvest earlier at lower sugar levels. In that case the grapes often lack physiological ripeness and have low varietal aroma contents $[13,25]$. Moreover unripe harvested grapes show often lower levels of yeast nitrogen and a higher tendency of atypical aging [26] The strategy of canopy management to delay ripeness by manipulating the leaf fruit ratio seems promising but can be risky as those treatments have to be done early in the growing season and the later weather conditions cannot be forecasted in such a long time frame.

A new promising solution for base wines with moderate and suitable alcohol levels could be the use of yeast that produce significantly less alcohol during the first fermentation. A critical point for the later wine quality and typicity is what substances are formed instead of the alcohols. The aim of this work was to evaluate if such commercial yeast that claim to produce less alcohol can be used for the elaboration of suitable base wines for sparkling wine production. 


\section{Material and methods}

The Pinot Noir grapes for the trials were harvested September 19th in 2017 with $88^{\circ}$ Oechsle and a sugar content of $202 \mathrm{~g} / \mathrm{l}$. The clarification was done by $18 \mathrm{~h}$ sedimentation and $30 \mathrm{mg} / \mathrm{l}$ of $\mathrm{SO}_{2}$ were added. No further fining products were added. The following day the musts were inoculated with $25 \mathrm{~g} / \mathrm{hl}$ of yeast. The first variant was fermented cool at $18{ }^{\circ} \mathrm{C}$ (control) and the second variant was fermented warm at $25^{\circ} \mathrm{C}$ (warm). Both variants were inoculated by Oenoferm Klosterneuburg from Erbslöh (Geisenheim, Germany). The third (W20) and the fourth (W21) variant were fermented at $18^{\circ} \mathrm{C}$ with two different commercial yeast strains that are claiming to produce less alcohol while producing more glycerol. The four different variants were fermented each in duplicate of 351 .

After fermentation the wines were racked and filtered by depth filtration with K 100 filter sheets (Pall, Bad Kreuznach, Germany). Previous to second fermentation, the wines were filtered by EK filter sheets (Pall, Bad Kreuznach, Germany). From each of the four base wine variants a lot of 251 was bottled in 0.751 bottles and closed with MCA crew caps. At the same time the second fermentation was started.

The sparkling wine production took place in 0.751 bottles according to traditional bottle fermentation.

The tirage was composed of:

- $24 \mathrm{~g} / \mathrm{l}$ sucrose

- $30 \mathrm{~g} / \mathrm{hl}$ Saccharomyces bayanus yeast (Lalvin DV 10, Lallemand, Canada)

- $50 \mathrm{~g} / \mathrm{hl}$ Vitamon Combi (Erbslöh, Geisenheim, Germany)

- $25 \mathrm{~g} / \mathrm{hl}$ Vitadrive F3 (Erbslöh, Geisenheim, Germany)

- $50 \mathrm{ml} / \mathrm{hl}$ Clarifant S (IOC, Mardeuil, France).

The fermentation and following storage of 9 month in total took place at cellar temperature of approximately $16-18^{\circ} \mathrm{C}$. The bottles were riddled by gyro palette and degorgement was done by freezing the bottle neck. Afterwards dosage was done by just adding $50 \mathrm{mg} / \mathrm{l}$ of $\mathrm{SO}_{2}$.

The base wine and the later sparkling wines were analyzed by NMR analyzer called Wine Screener (Bruker BioSpin GmbH, Rheinstetten, Germany).

The wine and sparkling wine analysis were done by NMR analyzer called Wine Screener (Bruker BioSpin $\mathrm{GmbH}$, Rheinstetten, Germany) at the Hochschule Geisenheim University by the Department of Beverage Research. The aroma analysis was done by the Department of Microbiology and Biochemistry according to Rapp et al. 1994. The method was modified by Fritsch, S, Brezina, S. and Rauhut, D. as follows:

Modification of the method:

Sample preparation:

$10 \mathrm{~mL}$ wine

$+\quad 2 \mathrm{~g} \mathrm{NaCl}$

$+10 \mu \mathrm{L}$ internal standard 2,6-dimethyl-5-hepten-2ol (DMH, $1219 \mu \mathrm{g} / \mathrm{L})$ for quantification control

$+10 \mu \mathrm{L}$ internal standard cumene $(168 \mu \mathrm{g} / \mathrm{L})$ as

$+160 \mu \mathrm{L} 1,1,2$-trichloro-1,2,2-trifluoroethane

20 minutes of shaking by Intelli-Mixer (NeoLab, Heidelberg/Germany)
Centrifugation with $3000 \mathrm{rpm}$ for 8 minutes (Megafuge 1.0, Heraeus Instruments, Hanau/Germany)

Injection volume $2 \mu \mathrm{L}$ sampling by glass pipette and drying of the extract by $\mathrm{Na}_{2} \mathrm{SO}_{4}(50 \mathrm{mg}$ on mineral wool)

Analytical parameter:

Injection system:

Thermal Desorption Unit KAS 3 (Gerstel, Mülheim an der Ruhr/ Germany)

Split less mode (1 min)

Start temperature: $30^{\circ} \mathrm{C}$, with $12^{\circ} \mathrm{C} / \mathrm{s}$ until $230^{\circ} \mathrm{C}$, 4 min stay

Gas chromatograph:

GC 5890 Series II, Hewlett Packard

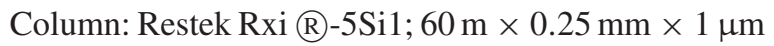

Carrier gas: helium

Solvent vent mode $10 \mathrm{~min}$

Flow: $0.8 \mathrm{~mL} / \mathrm{min}$

Start temperature: $40^{\circ} \mathrm{C}(5 \mathrm{~min})$

With $3{ }^{\circ} \mathrm{C} / \mathrm{min}$ until $125^{\circ} \mathrm{C}$, with $6^{\circ} \mathrm{C} / \mathrm{min}$ until $200^{\circ} \mathrm{C}$ (142 min)

Mass spectrometer:

5972 Mass Selective Detector, Hewlett Packard

Temperature: $180^{\circ} \mathrm{C}$

Mode: Scan (mass 35 until 250; 3,43 scans/sec).

The sensory analysis was carried out at after 9 month of bottle aging. Several triangle tests were conducted according to ISO 4120:2004 and ISO 8589:1988 to check if there is a significant difference between the four different variants of the base wine and the four different sparkling wine variants. A week later the same panel judged three base wines (control, W20 and W21) again according to quantitative descriptive analysis. In order to improve the homogeneity, the panel was trained to recognize and correctly use the selected attributes. Olfactory and gustatory standards were developed. The base wines were evaluated in triplicate according to a completely randomized design (Williams Latin Squares) to balance presentation order and carry over effect. The samples were presented in a monadic way and were served as $40 \mathrm{ml}$ samples in I.N.A.O. glasses of $210 \mathrm{ml}$ labeled with three-digit random numbers. The panelists scored each attribute on unstructured linear scales. The scales were anchored with the terms "low intensity" on the left and "high intensity" on the right. Data acquisition was assisted by FIZZ software (FIZZ network, v.2.38; Biosystème, Courtenon, France). The selected attributes were the following in Table 1.

The panelists were trained by wine aroma solutions. The basis for those standards was the control base wine spiked with respective aromas.

\section{Results and discussion}

The first fermentation showed no clear difference in terms of fermentation kinetics between the control and the warm fermentation. The two strains with lower alcohol production showed a similar fermentation behavior. All four variants, including the duplicates, fermented to dryness within 14 days. The second fermentation took place for all four variants with the same yeast strain of saccharomyces bayanus and showed slightly a different 


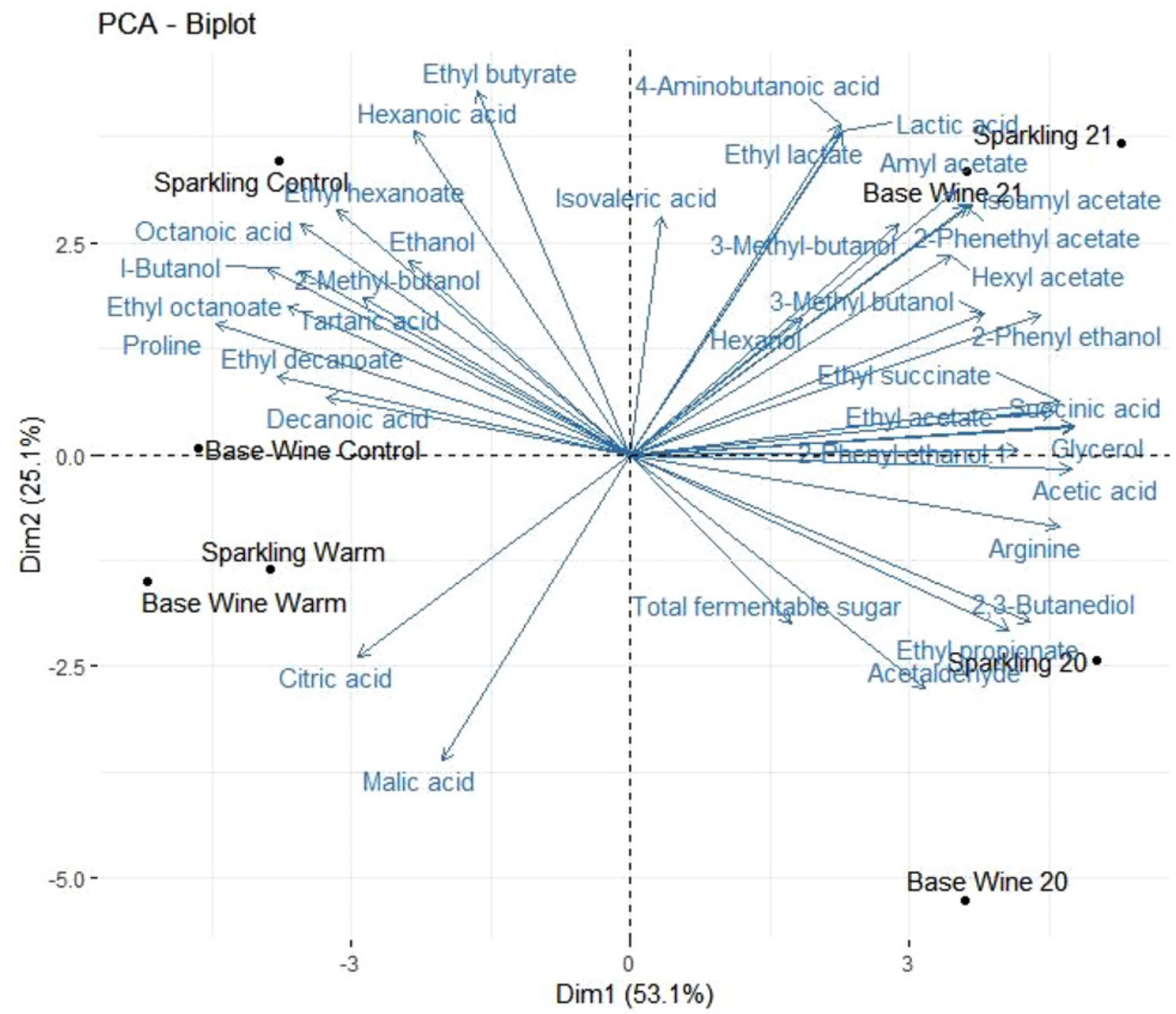

Figure 1. Principal component analysis (PCA) biplot showing difference in the aroma components measured by GC and the further components measured by NMR.

Table 1. List of selected attributes.

\begin{tabular}{|l|l|}
\hline Flowery & Aroma \\
\hline Cherry & Aroma \\
\hline Apricot & Aroma \\
\hline Citrus & Aroma \\
\hline Oxidative & Aroma \\
\hline Flowery & Flavor \\
\hline Cherry & Flavor \\
\hline Apricot & Flavor \\
\hline Citrus & Flavor \\
\hline Oxidative & Flavor \\
\hline Sweet & Flavor \\
\hline Bitter & Flavor \\
\hline Sour & Flavor \\
\hline Body/Fullness & Mouth feel \\
\hline
\end{tabular}

behavior. The control, in contrast to the other variants took a bit longer to build up pressure and the fermentation to dryness took consequently a bit longer. Nevertheless all sparkling wines fermented to levels of less than $0.5 \mathrm{~g} / \mathrm{l}$ of residual sugar.
The results of the different aroma components and further wine components measured by NMR and GC analysis in the base wines and the later sparkling wines show that the clearest influence is derived from the first fermentation and not that widely from the second fermentation in bottle. There is no clear tendency of the different yeasts to enhance a certain group of aroma components like esters or higher alcohols. But each base wine shows a certain individual aroma profile. The sparkling wines show a quite similar profile respective to the original base wines.

Figure 1 points out that there is a clear difference between the yeast with low alcohol yield (W20 and W21) towards the control and the warm variant in terms of proline and arginine. The samples W20 and W21 as well as the later sparkling wines, showed clearly reduced levels of proline. In the same time the arginine levels were significantly higher than that of the control. That is contradictory to the common point that proline is not reduced during fermentation [23] unless under aerobic conditions $[17,23]$. As the fermentation took place under common anaerobic conditions the reason could be the changed metabolism of the yeast. The elevated arginine content could be seen as a clear advantage for the second fermentation, as arginine is a major amino acid source for the yeast. 
Table 2. Results of the triangle tests * Different at a 95\% significance level (**99\%; ***99.9\%).

\begin{tabular}{|l|l|l|l|l|l|}
\hline Test Pair & $\begin{array}{l}\text { Answers } \\
\text { taken }\end{array}$ & $\begin{array}{l}\text { Answers } \\
\text { right }\end{array}$ & $\begin{array}{l}\text { Signif. } \\
\text { Risk }\end{array}$ & $\begin{array}{l}\text { Preference } \\
\text { ratio }\end{array}$ & $\begin{array}{l}\text { Signif. } \\
\text { Risk }\end{array}$ \\
\hline W Control/Wine Warm & 12 & 4 & 0.6069 & & \\
\hline W Control/W20 & 12 & 12 & $<0.0001^{* * *}$ & $11 / 1$ & $0.0063^{* *}$ \\
\hline W Control/W21 & 12 & 11 & $<0.0001^{* * *}$ & $9 / 1$ & $0.0215^{*}$ \\
\hline W20/W21 & 12 & 10 & $0.0005^{* * *}$ & $2 / 8$ & 0.1094 \\
\hline S Control/S Warm & 12 & 2 & 0.946 & & \\
\hline S Control/S20 & 12 & 8 & $0.0188^{*}$ & $5 / 3$ & 0.7266 \\
\hline S Control/S21 & 12 & 5 & 0.3685 & & \\
\hline S20/S21 & 12 & 2 & $0 ., 946$ & & \\
\hline
\end{tabular}

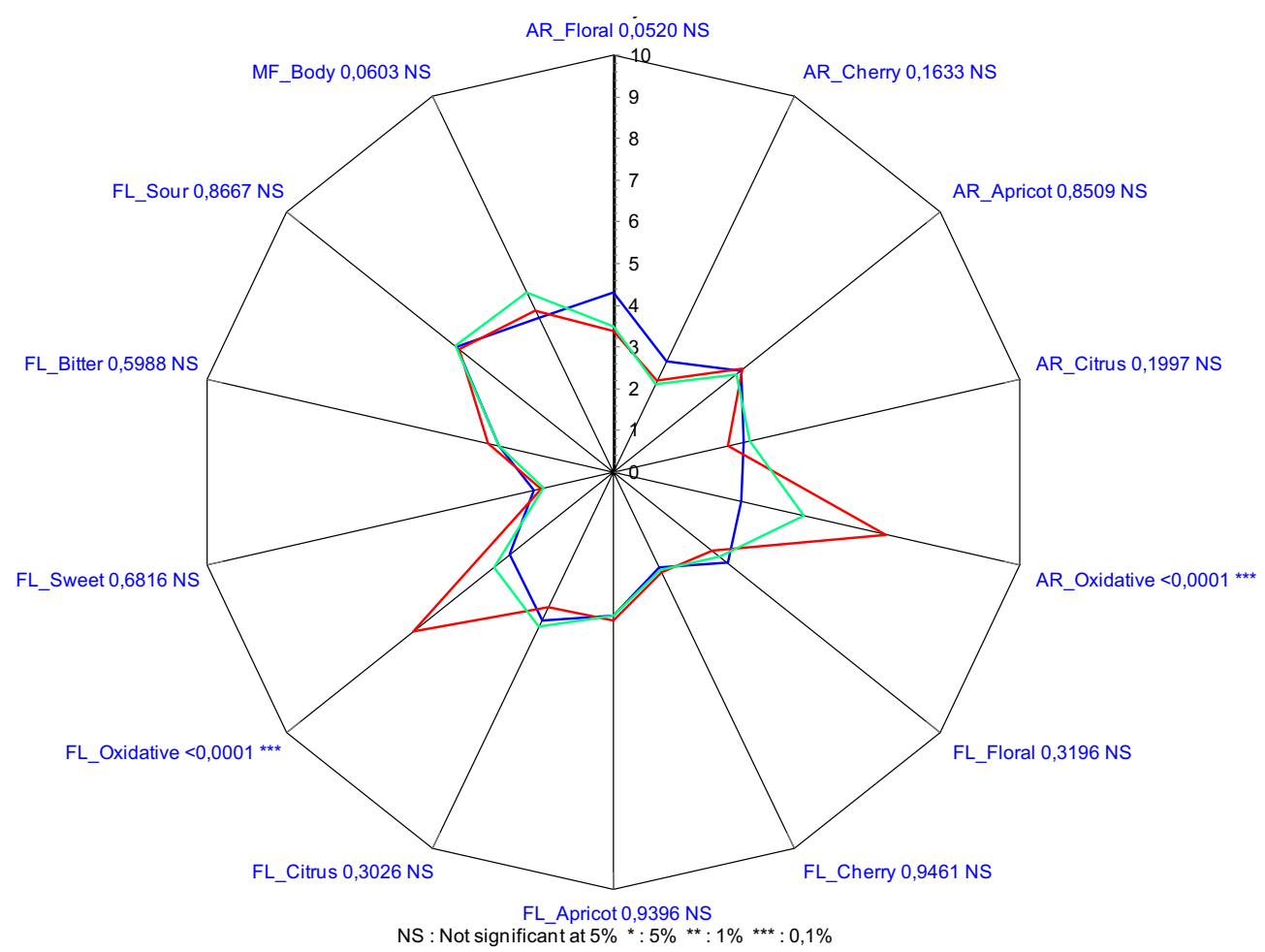

Figure 2. Graph of the mean sensory ratings of the 3 base wines (13 judges, 3 repetitions).

The glycerol content was as expected, significantly increased by the yeasts W20 and W21. W20 produced 3.5 and $\mathrm{W} 21$ produced $4.3 \mathrm{~g} / \mathrm{l}$ more glycerol than the control. As glycerol buffers the burning sensation of alcohol and gives the wine a certain body and fullness, it can be seen as the ideal antagonist and substitute of elevated alcohol contents.

Compared to the control, the variant W20 and W21 showed higher levels of total acidity. As the malic and tartaric acid values were similar for all variants, the levels of succinic acid were clearly increased by more than $0.3 \mathrm{~g} / \mathrm{l}$. The $\mathrm{pH}$ levels were nevertheless not significantly affected. As nowadays many regions lack more and more often acidity in their wines, the strategy to increase the acidity by fermentation appears very promising, especially in terms of targeted production of sparkling wine base wine.

On the other hand yeast W20 and W21 showed clearly increased levels of volatile acidity compared to the control. With values of 0.46 and $0.43 \mathrm{~g} / \mathrm{l}$ for the base wines and 0.53 respectively $0.49 \mathrm{~g} / \mathrm{l}$ the variants with reduced alcohol content had values around the sensory threshold [2]. The control variant showed with 0.2 respectively $0.25 \mathrm{~g} / \mathrm{l}$ in a uncritical value.

The alcohol yield was in case of the base wines with $47.3 \%$ for the control and $47.8 \%$ for the warm variant, in a typical range for saccharomyces cerevisiae. The variants W20 and W21 showed with 42.8 and $45.8 \%$ clearly reduced alcohol yields. The lower alcohol content at the same degree of residual sugar is validated due to the higher content of fermentation byproducts such as glycerol.

Table 2 shows the results of the triangle tests conducted by the panel in the first session. The panelists could not differ significantly the control base wine from the base wine fermented at $25^{\circ} \mathrm{C}$.

In the second triangle test the control wine was clearly different from the sample fermented with yeast W20 sample. Here all panelists could recognize the difference and clearly preferred the control towards the sample with less alcohol. This result was similar in the third triangle test where the panelists furthermore preferred significantly the control towards the sample resulting from a fermentation 
with finally less alcohol. The forth triangle test compared the two base wines with less alcohol. Here as well, the panelists could differ to a very high significance the samples from each other. In terms of preference the wine W21 was preferred more than the wine W20.

The comparison of the sparkling wines produced from the four different base wines was not as clear. Just the sparkling wine on basis of the control was differed in significant level from S20. The preference of the panelists was not clear in that case. The other tests did not show a clear significant differentiation.

The results indicate that the base wines aged within the 9 months more severe than the sparkling wines did age on the on the lees. A possibility could have been the relatively low sulfur content of $15 \mathrm{mg} / \mathrm{l}$ at bottling. Furthermore the lees and the autolysis derived aromas might have buffered sensorial differences coming from the base wine fermentation due to the different yeast strains and fermentation conditions.

Figure 2 shows the result of the second panel session. The tasters had to judge the base wines that showed clear significant differences in the first session. The variant fermented at $25^{\circ} \mathrm{C}$ did not differ significantly from the control. So it was not included in the quantitative descriptive analysis.

The previously selected attributes could not show clear significant differences in terms of the aroma and flavor descriptors, except oxidative characters. The control was significantly less oxidative in terms of smell and taste, compared to W20 and W21. An explanation for that elevated oxidative character can be found in the increased levels of acetaldehyde of the base wines W20 and W21. With more common levels of $\mathrm{SO}_{2}$, the base wines probably would have turned less oxidative. The body and fullness sensation of the sample W21 was slightly higher than the control. Not at a significant level. When taking into account that the alcohol content was clearly lower, it gets clear that the elevated glycerol production of $4.3 \mathrm{~g} / \mathrm{l}$ can substitute the alcohol in giving the wine a certain body sensation.

\section{Conclusion}

The trials show that new commercial yeast strain with lower alcohol yields could be an interesting option for the future to challenge generally rising alcohol levels and decreasing values of total acidity.

However, the increased levels of volatile acidity measured are an obstacle for the targeted sparkling wine production. It would be useful to work on the further optimization in terms of lower levels of volatile acid.

Further research should also investigate how the proline degradation could happen in case of the yeast with lower alcohol yield. That could deliver a clear advantage in terms of nutrient supply for the second fermentation.

\section{References}

[1] M.A. Amerine, E.B. Roessler, Wines: Their Sensory Evaluation (San Francisco: W.H. Freeman \& Co, 1983)

[2] H.P. Bach, G. Troost, O.H. Rhein, Sekt Schaumwein-Perlwein (Stuttgart, Ulmer, 2010)
[3] M. Bes, Les différentes stratégies microbiologiques et technologiques pour la production de vins à teneur réduite en alcool (Euroviti Congress, Montpellier, France, 2009)

[4] M. Bes, E. Aguera, V. Athes, A. Cadiere, P. Cottereau, S. Dequin, M. Mikolajczak, A. Roy, J.M. Sablayrolles, I. Souchon, A. Samson, J.L. Escudier, Revue des Oenologes 135, 9 (2010)

[5] N. Diban, V. Athes, M. Bes, I. Souchon, J. Membr. Sci. 311, 136 (2007)

[6] U. Fischer, A.C. Noble, Ame. J. Enology and Viticulture 45, 6 (1994)

[7] C. Garofalo, Fermentation 2, 21 (2016)

[8] R. Gawel, S. Van Sluyter, E.J. Waters, Aus. J. Grape and Wine Res. 13, 38 (2007)

[9] K. Grainger, Wine Quality: Tasting and Selection (John Wiley and Son's Ltd, Chichester/England, 2009)

[10] B.G. Green, Annals of the New York Acadamy of Science 510, 315 (1987)

[11] A.G.H. Lea, G.M. Arnold, J. Sci. Food and Agr. 29, 478 (1978)

[12] M.T. Lisanti, A. Gambuti, A. Genovese, P. Piombino, L. Moio, Food and Bioprocess Tech. 6, 2289 (2012)

[13] R. Longo, J.W. Blackmann, Food Chem. 261, 21 (2018)

[14] S. Martin, R.M. Pangborn, J. Sci. Food and Agr. 21, 653 (1970)

[15] D. Mattes, D. Meglio, Physiol. Behav. 72, 217 (2001)

[16] S. Meillon, D. Viala, M. Medel, C. Urbano, G. Guillot, P. Schlich, Food Quality and Preference 21, 732 (2010)

[17] Moreno-Arribas, Victoria M. Wine, Chemistry and Biochemistry (Springer, New York, 2009)

[18] A.C. Noble, Physiol. Behav. 56, 1251 (1994) Herausgeber:

[19] C. Nurgel, G.J. Pickering, J. Sensory Studies 21, 505 (2006)

[20] G.J. Pickering, D.A. Heatherbell, L.P. Vanhanen, M.F. Barnes, Amer. J. Enology and Viticulture 49, 306 (1998)

[21] Y. Ping, G.J. Pickering, Amer. J. Enology and Viticulture 59, 146-152

[22] G.J. Pickering, Physiol. Behav. 95, 4 (2008)

[23] P. Ribéreau-Gayon, D. Dubourdieu, B. Donèche, A. Lonvaud, Handbook of Enology (2.Auflage), Volume 1, The Microbiology of Wine and Vinifications, Chichester (John Wiley \& Sons Ltd, England, 2006)

[24] M. Schmitt, M. Christmann, Oenological / technical approaches to reduce elevated alcohol levels in wine (alcohol management) - sensory results, 35rd World Congress of Vine and Wine, Bucharest (Rumania/2013)

[25] M. Schmitt, Teilweise Alkoholreduzierung von Wein mittels physikalischer Verfahren - Alkoholmanagement, Dissertation (Hochschule Geisenheim University, 2016)

[26] U. Schneider, Am. J. Enol Vitic. 65, 277 (2014)

[27] A. Scinska, Drug Alcohol Dependence 60, 199 (2000) 
[28] C. Urbano, (2007) R-index and triangular tests to determine the perception threshold of a reduction of alcohol content in wine, 7th Urbano, C.; Dupressoir, C.; Samsom, A.; Cordelle, S. and Guillot, G. (2007). R-index and triangular tests to determine the perception threshold of a reduction of alcohol content in wine, 7th Pangborn Sensory Science Symposium, Minneapolis. USA. 12-16
[29] D. Wollan, The right tools for the job: dealing with cool climate wine styles. Proceedings of the sixth international symposium for cool climate viticulture and enology (New Zealand Society for Viticulture and Enology, Auckland, 2006), p. 159

[30] M.C. Zamora, M.C. Goldner, M.V. Galmarini, J. Sens. Stud. 21, 601 (2006) 